\title{
Putting co-sleeping into perspective
}

\author{
Peter S. Blair*
}

\begin{abstract}
Co-sleeping begins in infancy and often continues through to childhood as part of early nurturing practices. In evolutionary terms the human infant is the most neurologically immature primate at birth and develops the most slowly, thus intense and prolonged mother-infant contact acts as a protective mechanism against physiological difficulties and environmental assaults. Proposed benefits of close contact between infants and caregivers include improved cardiorespiratory stability and oxygenation, fewer crying episodes, better thermoregulation, an increased prevalence and duration of breastfeeding, and enhanced milk production. ${ }^{1,2}$ The postulated benefits of breastfeeding are increasingly being recognised and actively promoted by the
\end{abstract} World Health Organization and United Nations Children's Fund. Co-sleeping can facilitate breastfeeding and the promotion of one practice can lead to the promotion of the other. However, the parental bed is not designed with infant safety in mind and co-sleeping has been implicated in rare but fatal accidental infant deaths due to entrapment and parental overlaying. More recently epidemiological studies investigating sudden infant death syndrome (SIDS) have shown a proportional increase of these deaths in the co-sleeping environment ${ }^{3}$ which has led some authorities, including the American Academy of Pediatrics ${ }^{4}$ to recommend against bed-sharing. The unusual level of criticism and hostility ${ }^{5-8}$ generated by this recommendation is a testament to the current polarised debate both within and beyond the field of SIDS of the potential risk and perceived benefits of parents and infants sharing the same bed. As Santos et al. ${ }^{9}$ perceptively point out in their investigation of this infant care practice in Brazil, the advantages and risks are perceived in accordance with the values of a given society.

In a society where co-sleeping is rare or mainly associated with ethnic minority groups or low income families it is perhaps easier to label such a practice as a risk factor in itself. SIDS is also described as "cot death" because this is the environment in which a lot of these infants are found. However, the "cot" as an infant sleep environment has never been treated as a risk factor in SIDS research but rather closer attention has been given to the particular circumstances within the cot that may confer risk or protection to the infant. In contrast, co-sleeping is perceived to be a risk factor and few studies have taken into account the specific circumstances in which these young infants die such as whether the bed was actually a sofa or whether the parent had consumed too much alcohol or sleep-inducing drugs. Treated in this rudimentary way, lumping together clearly inappropriate and dangerous rare events with accepted mother-infant care interactions and ascribing simplistic labels such as "safe" or "unsafe" to the whole, there is ample evidence to advise against such practice. If society perceives no benefit to co-sleeping then there is perhaps merit to this approach as nobody contests the fact that the cot beside the parental bed is the safest place for the infant to sleep. Simply advising against co-sleeping, however, does assume that parents will listen to such advice and it also assumes that parents have a choice in the matter. Most young infants commonly wake during the night and most need to be fed, occasionally mothers will fall asleep when doing so, in which case the parental bed is a far safer option than a chair or sofa. Telling mothers not to co-sleep precludes specific advice on how to do this safely, ignores cultural preference and reduces the options of where mothers can feed their infants during sleep.

In many societies co-sleeping is not rare. In England almost half of all neonates bed-share at some time with their parents, $1 / 5$ of infants are brought into the parental bed on a

* Senior Research Fellow, Institute of Child Life \& Health, University of Bristol, Bristol, UK.

No conflicts of interest declared concerning the publication of this editorial.

Suggested citation: Blair PS. Putting co-sleeping into perspective. J Pediatr (Rio J). 2008;84(2):99-101

doi:10.2223/JPED.1775 
regular basis over the first year of life. ${ }^{10}$ Similar or higher rates of bed-sharing at 3 months of age have recently been reported in other European countries; Ireland (21\%), Germany (23\%), Italy (24\%), Scotland (25\%), Austria (30\%), Denmark $(39 \%)$, Sweden $(65 \%) .{ }^{11}$ Even in countries where bed-sharing is uncommon such as Holland, Norway and the USA, all have reported an increase in the prevalence of bed-sharing in the last decade. ${ }^{3}$ This is at a time when breastfeeding rates have also risen. In Brazil, Santos et al. report that co-sleeping is common amongst 1 -year-old infants $(46 \%)$, especially amongst the younger mothers, less educated mothers and those with lower socioeconomic status. ${ }^{9}$ Similar findings have been reported in the USA and New Zealand but the perception that co-sleeping is somehow an undesirable infant care practice because it is related to the more deprived groups is not necessarily an accurate one. In Brazil, breastfeeding is common amongst the poorest groups and more common amongst intermediary socioeconomic groups than those with the highest status, but is seen as a desirable practice that is encouraged as being positive for both the mother and infant. ${ }^{12}$ The use of pacifiers is commonly associated with poorer socioeconomic groups but is a practice that is now being encouraged as a potential protective mechanism against SIDS or at least a marker of something the parents seem to be doing right. ${ }^{13}$ Deprivation does not necessarily equate to poor infant care practices and neither is co-sleeping the preserve of the more deprived sections of the population. In England co-sleeping straddles the social boundaries $^{10}$ as it does in Sweden ${ }^{14}$ and several non-Western cultures.

In certain cultures bed-sharing is the common infant care practice and the SIDS prevalence is high. These include the black populations in the USA and the Maori and aboriginal populations in the Southern Hemisphere. Intriguingly, however, there are other cultures where bed-sharing is also the common practice but the SIDS rates are quite low, including Japan and Hong Kong, the Bangladeshi and Asian communities in the UK and Pacific Islander communities in New Zealand. ${ }^{3}$ It is not bed-sharing that distinguishes these cultures but there are other mediating factors such as maternal smoking, which is particularly low in Japan and Hong Kong ${ }^{11}$ and parental alcohol consumption, which is higher amongst the Maori and Aboriginal populations ${ }^{15,16}$ that may combine with co-sleeping and play a role in SIDS deaths. Another mediating factor might be the sleeping environment itself, the Japanese futon for instance, a firm thin mattress placed on the floor is intrinsically different from the elevated often softer mattresses used in Western societies.

Far fewer epidemiological studies have investigated potential benefits associated with infant care practices than have investigated practices possibly linked with infant morbidity and mortality. The interpretation of the findings and indeed how the questions are phrased and responses delineated, can be difficult to work out and again dependent on how different societies perceive such practices. Santos et al. have shown that infant night waking is associated with co-sleeping. ${ }^{9}$ One interpretation is that the presence of an adult may disturb the sleeping infant causing them to wake, another is that co-sleeping is a consequence, a solution if you will, to the fact the infant keeps waking in the night. A third possibility hinted at by the authors is that infant waking is being perceived differently by co-sleeping mothers as perhaps a more positive interaction associated with the necessity of breastfeeding. Thus, although incessant night time waking can, quite rightly, be described as a sleeping disorder, frequent waking can also be described as a hungry baby who the mother is happy to feed. Devising the questions for the mothers to articulate these differences is perhaps more complex than constructing crude risk factors for morbidity studies.

Some co-sleeping environments, especially with young infants, are clearly dangerous and parents need to be made aware what these circumstances are and how to avoid them. The potential immediate and future benefits of infants and parents sharing the same sleeping surface also need to be studied. To do this properly we perhaps all need to leave behind our own culturally determined pre-conceptions and look more closely at the specific circumstances in which co-sleeping occurs, the changing parental decision making that determines this practice over time and the experience of both those who choose to co-sleep and those who do not.

\section{References}

1. Anderson GC. Current knowledge about skin-to-skin (kangaroo) care for preterm infants. J Perinatol. 1991;11:216-26.

2. Ludington-Hoe SM, Hadeed AJ, Anderson GC. Physiological responses to skin-to-skin contact in hospitalized premature infants. J Perinatol. 1991;11:19-24.

3. Blair PS, Fleming P. Co-sleeping and infant death. In: David TJ, editor. Recent advances in Pediatrics 24. London: Royal Society of Medicine Press; 2007.

4. American Academy of Pediatrics, Task Force on Sudden Infant Death Syndrome. The changing concept of sudden infant death syndrome: diagnostic coding shifts, controversies regarding the sleeping environment, and new variables to consider reducing the risk. Pediatrics. 2005;116:1245-55.

5. Gessner BD, Porter TJ. Bed sharing with unimpaired parents is not an important risk factor for sudden infant death syndrome. Pediatrics. 2006;117:990-1; author reply 994-6.

6. Eidelman AI, Gartner LM. Bed sharing with unimpaired parents is not an important risk factor for sudden infant death syndrome: to the editor. Pediatrics. 2006;117:991-2; author reply 994-6.

7. Bartick M. Bed sharing with unimpaired parents is not an important risk factor for sudden infant death syndrome: to the editor. Pediatrics. 2006;117:992-3; author reply 994-6.

8. Pelayo R, Owens J, Mindell J, Sheldon S. Bed sharing with unimpaired parents is not an important risk factor for sudden infant death syndrome: to the editor. Pediatrics. 2006;117:993; author reply 994-6. 
9. Santos IS, Mota DM, Matijasevich A. Epidemiology of co-sleeping and nighttime waking at 12 months in a birth cohort. J Pediatr (Rio J). 2008;84(2):114-22

10. Blair PS \& Ball HL. The prevalence and characteristics associated with parent-infant bed-sharing in England. Arch Dis Child. 2004; 89:1106-10.

11. Nelson EA, Taylor BJ. International Child Care Practices Study: infant sleeping environment. Early Hum Dev. 2001;62:43-55.

12. Araujo CL, Victora CG, Hallal PC, Gigante DP. Breastfeeding and overweight in childhood: evidence from the Pelotas 1993 birth cohort study. Int J Obes (Lond). 2006;30:500-6.

13. Mitchell EA, Blair PS, L'Hoir MP. Should pacifiers be used to prevent sudden infant death syndrome? Pediatrics. 2006;117:1755-8.

14. Welles-Nystrom B. Co-sleeping as a window into Swedish culture: considerations of gender and health care. Scand J Caring Sci. 2005;19:354-60.

15. Chikritzhs T, Brady M. Fact or fiction? A critique of the National Aboriginal and Torres Strait Islander Social Survey 2002. Drug Alcohol Rev. 2006;25:277-87.
16. Scragg R, Mitchell EA, Taylor BJ, Stewart AW, Ford RP, Thompson $\mathrm{JM}$, et al. Bed sharing, smoking, and alcohol in the sudden infant death syndrome. New Zealand Cot Death Study Group. BMJ. 1993;307:1312-8.

Correspondence:

Peter Blair

FSID Research Unit, Level D

Southwell St., St. Michaels Hospital

BS2 8EG - Bristol - UK

Tel.: +44(117) 928.5145

Fax: +44 (117) 928.5154

E-mail: p.s.blair@bris.ac.uk

\section{Sleep-disordered breathing in children: time to wake up! \\ Oliviero Bruni*}

The scientific literature in the last few decades stressed the importance of identifying and treating sleep-disordered breathing (SDB) in children, since it might determine serious neurobehavioral, cardiovascular, endocrine, and metabolic consequences.

Accurate identification of the prevalence of primary snoring and obstructive sleep apnea (OSA) in the pediatric population is critical for different reasons: a) estimate the magnitude of the problem; $b$ ) inference on the relationships with other emergent childhood health problems, such as obesity; c) possibility of preventing long-term consequences; d) identification of population subgroups that could be at risk for developing SDB; and e) guide for future investigations.
One of the first worries of medical research when approaching a health problem is to clearly identify its magnitude, to evaluate how many subjects could be affected and how health related diseases could be prevented. This is increasingly more important if we care about children and if we are aware that we could avoid several long-standing consequences with early treatment of sleep breathing problems.

In this perspective, the very well conducted study of Petry et al. ${ }^{1}$ in this issue of Jornal de Pediatria is of great importance because it gives for the first time a clear picture of SDB problems in children in a Brazilian region. This cross-sectional study was carried out in the city of Uruguaiana, RS, in a large sample (about

* Pediatric Sleep Center, Sapienza University, Rome, Italy.

No conflicts of interest declared concerning the publication of this editorial.

Suggested citation: Bruni O. Sleep-disordered breathing in children: time to wake up! J Pediatr (Rio J). 2008;84(2):101-103

doi: $10.2223 / 7557$ 